\title{
Emergency Cardiac Surgery in Patients on Oral Anticoagulants and Antiplatelet Medications
}

\author{
Rami Akhrass ${ }^{1}$, A. Marc Gillinov ${ }^{1}$, Faisal Bakaeen ${ }^{1}$, Deena Akras ${ }^{1}$, Scott Cameron ${ }^{1}$, Jay \\ Bishop $^{1}$, Samir Kapadia ${ }^{1}$, and Lars Svensson ${ }^{2}$ \\ ${ }^{1}$ Cleveland Clinic \\ ${ }^{2}$ Cleveland Clinic Main Campus Hospital
}

May 23, 2021

\begin{abstract}
Emergency surgery, blood transfusion, and reoperation for bleeding have been associated with increased operative morbidity and mortality. The recent increased use of direct oral anticoagulants and antiplatelet medications have made the above more challenging. In addition, cardiopulmonary bypass $(\mathrm{CPB})$ with its associated hemodilution, fibrinolysis and platelet consumption may exacerbate the pre-existing coagulopathy and increase the risk of bleeding. Management decisions are typically made on a case-by-case basis. Surgery is delayed when possible and less invasive percutaneous options should be considered if feasible. Attention is paid to exercising meticulous techniques, avoiding excessive hypothermia and treating coexisting issues such as sepsis. Ensuring a dry operative field upon entry by correcting the coagulopathy with reversal agents is offset by the concern of potentially hindering efforts to anticoagulate the patient (heparin resistance) in preparation for CPB, in addition to possibly increasing the risk of thromboembolism. Proper knowledge of the anticoagulants, their reversal agents, and the usefulness of laboratory testing are all essential. Platelet transfusion remains mainstay for antiplatelet medications. Four-factor prothrombin complex concentrate is considered in patients on oral anticoagulants if CPB needs to be instituted quickly. Specific reversal agents such as idarucizumab and andexanet alfa can be considered if significant tissue dissection is anticipated such as redo sternotomy, but are costly and may lead to heparin resistance and anticoagulant rebound.
\end{abstract}

\section{Emergency Cardiac Surgery in Patients on}

\section{Oral Anticoagulants and Antiplatelet Medications}

Rami Akhrass, MD, ${ }^{1}$ Marc Gillinov, MD, ${ }^{1}$ Faisal Bakaeen, MD, ${ }^{1}$ Deena Akras, ${ }^{1}$ Scott $\mathrm{J}$. Cameron, MD, PhD, ${ }^{2}$ Jay Bishop, MD, ${ }^{2}$ Samir Kapadia, MD, ${ }^{2}$ and Lars Svensson, MD, $\mathrm{PhD}^{1}$

1 Department of Thoracic and Cardiovascular Surgery, ${ }^{2}$ Department of Cardiovascular Medicine, Heart, Vascular and Thoracic Institute, Cleveland Clinic, Cleveland. Ohio.

Funding: There was no financial support for this study.

Disclosures: All authors declare no conflicts of interest except: Dr. Bishop is on speaker bureau for Janssen and Dr. Gillinov is consultant to Edwards Lifesciences, Medtronic, Abbott, CryoLife, AtriCure, Johnson and Johnson, ClearFlow and has the right to equity from ClearFlow.

Word count: 3,398

Address for correspondence:

Rami Akhrass, MD

Department of Thoracic and Cardiovascular Surgery 
Heart, Vascular and Thoracic Institute

9500 Euclid Avenue

Cleveland, Ohio 44195

akhrasr@ccf.org

Central Message:

Emergency cardiac surgery in patients on oral anticoagulants and antiplatelet medications is challenging. Knowledge of the anticoagulants and their reversal agents with their limitations is essential.

Central picture:

Oral anticoagulants and antiplatelet medications with their reversal agents

Keywords:

Direct oral anticoagulants-antiplatelets-bleeding-transfusion-coagulopathy-emergency cardiac surgery.

\section{Introduction:}

Bleeding is a major concern in cardiac surgery with possibly requiring blood transfusions and re-exploration, both identified as independent risk factors for increased perioperative morbidity and mortality, especially when intervening in an emergency setting. ${ }^{1-4}$ The risk of bleeding is amplified in patients on oral anticoagulants and antiplatelet medications that have become a cornerstone in the treatment of conditions, such as strokes, coronary artery disease (CAD), atrial fibrillation $(\mathrm{AF})$, peripheral vascular disease, and deep venous thrombosis (DVT). ${ }^{5,6}$

Over 6-million patients are on anticoagulants and this number is expected to rise to 12-million in 2030, mainly due to the aging population with an increased prevalence of AF, and to the earlier diagnosis of occult AF with use of implantable loop recorders. ${ }^{5,7}$ Direct oral anticoagulants (DOACs), such as direct thrombin inhibitor (dabigatran) and direct factor Xa inhibitors (rivaroxaban, apixaban, edoxaban, betrixaban), have become first-line choices in the treatment and prevention of thromboembolism as well as stroke prophylaxis in patients with AF. ${ }^{6}$ Randomized trials have demonstrated the superiority of dabigatran (RE-LY trial) and apixaban (ARISTOTLE trial) over the traditionally used vitamin-K antagonists (VKAs) in thromboembolic stroke prevention. ${ }^{8,9}$ In addition, DOACs have several other advantages including, rapid onset in their action limiting the need for bridging, less drug-drug and drug-food interactions, and easier use with reduction of blood level monitoring.

Anticoagulants also include antiplatelet medications, such as aspirin (ASA) and the $\mathrm{P} 2 \mathrm{Y}_{12}$ receptor antagonists (clopidogrel, ticagrelor and prasugrel), used for the treatment of CAD, acute coronary syndrome (ACS) and following percutaneous coronary interventions (PCI). Patients frequently receive dual antiplatelet medications consisting of ASA and a $\mathrm{P}_{2} \mathrm{Y}_{12}$ receptor antagonist, shown to improve survival and reduce in-stent thrombosis. ${ }^{10}$

The above landscape has made operating on patients receiving oral anticoagulants and antiplatelet medications inevitable and an unfortunate reality. We sought to review available literature with regards to guidelines on management of patients receiving above medications who require emergency cardiac operations. Informed consent and institutional review board approval were not required and were waived for the purpose of this study.

\section{Value and limitations of laboratory testing:}

Examination of the patient's coagulation and hematological profiles is critical; however; treatment options hinge upon proper knowledge of the usefulness of various laboratory testing available. Normal laboratory values are usually reassuring but may not always translate into clinical hemostasis. It is helpful to know the mechanism of action of the drug at hand and its effect on the coagulation cascade to develop an understanding 
of the true value and usefulness of various blood tests (Figure 1). For example, the international normalized ratio (INR) was developed to monitor VKAs and has limited value in DOACs. ${ }^{11}$

The degree of anticoagulation and drug levels are of paramount importance in determining the course of action. Typically, the anticoagulant washes out after 5 half-lives have elapsed, taking into account the patient's underlying renal and hepatic functions (Table1). ${ }^{12}$ Main advantages of DOACs include their rapid onset of action (Cmax in most cases is 1-4 hours) and short half-lives (9-15 hours, with the exception of betrixiban of 37 hours). In addition, the pharmacodynamic effect of this class of anticoagulants is even shorter, unlike warfarin that has a much longer pharmacodynamic, compared to its pharmacokinetic, halflife. Thus, urgent reversal of DOAC agents may not always be necessary; however, in emergency cases, monitoring of DOAC levels might be helpful, with several important considerations to take note of when evaluating laboratory values:

-Prothrombin time (PT) : There is little correlation between PT level and DOACs. ${ }^{11,13}$ PT is more reliable with higher dabigatran levels. ${ }^{13}$

-Thrombin time (TT): A normal TT virtually excludes clinically relevant dabigatran levels; however, subtherapeutic levels of dabigatran may prolong TT and is most useful as a quantitative tool. ${ }^{11}$

-Dilute thrombin time (dTT): measured by hemocolt, correlates well with dabigatran levels and decreases the sensitivity of TT test by diluting the patient's sample with normal plasma. ${ }^{14}$

-Liquid chromatography or (Tandem mass spectrometry) : is the gold standard for assessing DOAC concentration; however, there is minimal clinical outcome data and the test may not be widely available in clinical settings. ${ }^{11,15}$

-Ecarin Clotting Time: This clotting assay uses ecarin derived from the saw-scaled viper and is used to measure the activity of direct thrombin inhibitors such as dabigatran. This test relies on both the prothrombin and fibrinogen in the patient's sample and if abnormal can result in issues with interpretation. The Ecarin Chromogenic Assay is independent of variability in prothrombin and/or fibrinogen levels and is not influenced by VKA. ${ }^{16}$

-Anti-Xa activity : The absence of anti-factor Xa activity excludes any clinically significant drug levels, but the test may not be widely available. ${ }^{17}$

-Thromboelastography (TEG) or Rotational thromboelastometry (ROTEM) : Thrombin is generated in the TEG or ROTEM sample cups and may produce a false normal test despite the presence of clinical coagulopathy. TEG and ROTEM may not detect platelet defect due to ASA, dipyridamole or P2 $\mathrm{Y}_{12}$ receptor antagonists. A dose-dependent increase in ROTEM clotting time was noted for DOACs and low levels of edoxaban, rivaroxaban or dabigatran only poorly impacted ROTEM tests. Apixaban had only a low effect even at high concentrations. ${ }^{18}$

-Quantra QPlus : A novel point-of-care platform that uses ultrasonic pulses to characterize dynamic changes in viscoelastic properties of a blood sample during coagulation. In a prospective study, the Quantra predicted clinical need for platelet transfusion but not for fresh frozen plasma (FFP). ${ }^{19}$

\section{Management:}

Several recommendations can be made concerning the acute management of coagulopathy in patients undergoing emergent cardiac surgical intervention depending on the anticoagulant and are summarized in (Table 2).

1-Activated oral charcoal is given if DOAC was last ingested within 2-4 hours.

2-Vitamin K (5-10 mg IV) is administered for VKA reversal; however, its effect may be too slow for emergent surgery. ${ }^{20}$ 
3-Non-specific prohemostatic agents can be used including four-factor prothrombin complex concentrate (4FPCC) with a recommended dose of $25-50 \mathrm{IU} / \mathrm{kg}$. Four-factor PCC was found to be close to $70 \%$ effective in achieving hemostasis in patients with major bleeding. ${ }^{21,22}$ In a review by Goldstein et al, 4 F-PCC was superior to FFP for rapid INR reversal and effective hemostasis in patients requiring VKA reversal for urgent surgical procedures (most were non-cardiac). ${ }^{23}$ One study reported 40 patients on oral VKA, mainly for AF, who underwent urgent cardiac surgery and were randomized to FFP and PCC. Patients received, pre cardiopulmonary bypass (CPB), either 2 units of FFP or half of the calculated PCC dose. Post-bypass, they received 2 more units of FFP or the second half of PCC dose. It was noted that reversal with PCC was faster and bleeding was less compared to FFP. ${ }^{24}$

4- Three-factor PCC has less concentration of factor VII compared to 4F- PCC and can be used for reversal of VKA. Both 4F-PCC and 3F-PCC do not require large volumes to be administered, an advantage in patients with heart failure and fluid overload.

5-Antifibrinolytic agents such as aminocaproic acid and tranexamic acid are frequently used even in elective cases. They exert their effect by inhibiting plasminogen activators and to a lesser degree through antiplasmin activity, thereby minimizing the untoward effects of fibrinolysis seen with CPB and challenging an already attenuated hematologic profile. ${ }^{25}$

6-Two specific DOAC reversal agents (antidotes) have been approved by the US Food and Drug administration (FDA): idarucizumab for reversal of dabigatran and andexanet alfa for reversal of apixaban, edoxaban and rivaroxaban. ${ }^{26}$ Dosing is typically based on predicted concentration of the anticoagulant depending on its dose and last time of ingestion.

Idarucizumab was studied in patients with dabigatran-associated bleeding (REVERSE-AD trial). Most common sites of bleeding were gastrointestinal and intracranial. Median time to achieve hemostasis was 2.5 hours and there was no correlation with drug levels. None underwent CPB and several patients suffered thromboembolic events, $50 \%$ of which occurred within 5 days. ${ }^{27}$ In a multicenter experience in Spain, Idarucizumab was used in 56 heart transplant patients, $7.5 \%$ required reoperations for bleeding and $66 \%$ were transfused with blood products. ${ }^{28}$

The use of andexanet alfa (a recombinant modified factor Xa protein) as an antidote was reported on 352 patients on factor Xa inhibitors who had major bleeding (64\% intracranial and $26 \%$ gastrointestinal). It markedly reduced anti-factor Xa activity with good or excellent hemostatic effects in $82 \%$ patients. ${ }^{29}$ In one prospective study of 66 patients on factor Xa inhibitors and presenting with major bleeding, reversal was effective in $68 \%$, ineffective in $32 \%$ and $8 \%$ suffered a major thromboembolic event. ${ }^{30}$

These drugs are costly and have a short half-life that may result in anticoagulant rebound when infusion is stopped. On the other hand, specifically andexanet alfa, can bind to heparin-antithrombin complexes precluding therapeutic activated clotting time (ACT) necessary for CPB (heparin resistance) and should be used with caution. ${ }^{31-33}$ It is worthwhile reporting on a potential promising universal antidote (ciraparantag) to all DOACs that binds to the anticoagulants via noncovalent hydrogen bonds, but is still under investigation and not available for commercial use ${ }^{34}$ (Table 3 ).

7-Hemodialysis (HD) is thought not to be very beneficial with regards to urgent reversal as most DOACs, including rivaroxaban and apixaban, are highly protein bound. Although edoxaban is relatively low protein bound, it is not well cleared by HD. Betrixiban is $60 \%$ protein bound, but there is paucity of data demonstrating the effectiveness of dialysis in clearing the drug. Dabigatran is $35 \%$ protein bound and HD has been proposed as a possible treatment strategy in patients with major bleeding. Published reports have shown that 4-hours of HD can reduce the plasma concentration of dabigatran by up to $68 \%$, with a recent meta-analysis suggesting HD may be of some benefit in patients requiring urgent reversal. ${ }^{35}$

8-Antiplatelet medications: Aspirin irreversibly acetylates a serine residue of cyclooxygenase 1, which ultimately decreases the synthesis and release of the platelet-activating molecule thromboxane A2 $\left(\mathrm{TxA}_{2}\right)$. The aspirin effect is permanent and lasts for the lifespan of the circulating platelet (7-10 days) ${ }^{36}$ In was noted 
that there was no increased risk of mortality or bleeding among patients undergoing coronary artery bypass grafting (CABG) perioperatively taking ASA compared with placebo, but it was associated with increased transfusion requirements. ${ }^{37,38}$ Oral $\mathrm{P} \mathrm{Y}_{12}$ receptor antagonists, such as clopidogrel, prasugrel and ticagrelor, can be more problematic given their more potent antiplatelet effect. Like aspirin, the thienopyridine P2 $\mathrm{Y}_{12}$ receptor antagonists clopidogrel and prasugrel covalently modify the receptor and the antiplatelet effect lasts for the lifespan of the platelet. Ticagrelor; however, is a non-thienopyridine $\mathrm{P} 2 \mathrm{Y}_{12}$ receptor antagonist and its antiplatelet effect lasts for approximately 4 days. In patients undergoing urgent CABG with prior ticagrelor administration, serial platelet aggregometry revealed a return to normal platelet function within 3-days. ${ }^{39}$

The intraoperative use of Cytosorb adsorption, previously used in septic patients, was tested in Europe on 55 patients who were on ticagrelor or rivaroxaban and required emergency cardiac surgery, mostly CABG. The Cytosorb adsorpter is included in the CPB circuit between the oxygenator and the venous reservoir. The filter is made of biocompatible porous beads capable of removing substances based on pore capture and surface adsorption. It was effective in reducing bleeding complications with the majority of patients not requiring transfusions and none required re-exploration (compared to $37.5 \%$ in the non-Cytosorb group). ${ }^{40}$ The FDA has issued an Emergency Use Authorization for the use of Cytosorb to treat COVID-19 patients and respiratory failure; however, further testing to corroborate the findings in the European study and determine its effect on other substrates such as albumin and antibiotics will be important.

\section{Discussion:}

The deleterious effects of blood transfusions are well documented and calls have been made to restrict their use. In a study of greater than 18,000 patients at The Cleveland Clinic over 10-years, it was noted that transfusions and reoperations for bleeding were independently associated with increased risk of morbidity and mortality ( $8.5 \%$ vs $1.8 \%)^{2}$ Similarly, others showed a higher mortality ( $14.2 \%$ vs $\left.3.4 \%\right)$ in patients requiring re-exploration compared to the rest, and the amount of packed red blood cells (pRBC) was associated with an incremental increased risk ( $0.25 \%$ for each unit transfused) ${ }^{41}$ In another study of almost 5,400 patients, those who required re-exploration for bleeding had a twofold mortality increase in early postoperative period, as well as an overall mortality increase beyond 90 days. ${ }^{42}$ In addition, a meta-analysis review of observational studies showed that preoperative exposure to clopidogrel was associated with increased risk of death, blood loss, transfusions and reoperations. ${ }^{44}$ An individualized strategy for clopidogrel suspension was suggested in patients undergoing CABG following ACS, guided by platelet function testing, which significantly reduced postoperative bleeding and blood use. ${ }^{45}$

A review of 528,686 CABG patients, from the Society of Thoracic Surgery (STS) database, revealed that patients requiring emergent surgery or were on antiplatelet medications within 24-hours before the operation had a significantly higher rate of re-exploration for bleeding, with an increased risk-adjusted mortality of $5.9 \%$ compared to $2.0 \%$ for others. ${ }^{43}$ Likewise, a meta-analysis of 557,923 patients found emergency surgery and preoperative aspirin use were among the risk factors resulting in higher rate of re-exploration with an increased risk of mortality and major morbidity. ${ }^{1}$ In a separate study, it was noted that patients who received clopidogrel prior to urgent CABG had a higher rate of re-exploration and blood transfusions. ${ }^{47}$ Finally, an international prospective study of patients undergoing urgent CABG on antithrombotic agents (ACUITY trial) noted that transfusions of [?] 4 units pRBC was an independent mortality risk factor for up to one year post surgery. ${ }^{46}$

The decision process is easier in patients for whom surgery can be done semielectively or at least delayed for adequate anticoagulant washout, as per the guidelines set forth by the STS. ${ }^{48}$ Patients with high risk for thromboembolism can be bridged on short half-life medications such as heparin that can be quickly reversed. Patients with multi-vessel CAD on anticoagulants presenting with ST-elevation myocardial infarction (STEMI) would undergo PCI of the culprit vessel and surgical revascularization delayed until adequate anticoagulant washout.

However, it is inevitable that surgeons will be confronted where emergency surgery is needed for patients on 
anticoagulants. Examples would be a patient with ACS where PCI in not possible due to coronary anatomy or lack of expertise, or a patient with an aortic dissection, where the risk of bleeding is further aggravated by coagulopathy induced by hypothermia and circulatory arrest, resulting in platelet dysfunction and reduced activity of clotting factors. ${ }^{49}$ Other examples include endocarditis and sepsis, where underlying issues, such as dilution, hemolytic anemia and disseminated intravascular coagulopathy (DIC) worsen the coagulopathy. In addition, CPB creates its own insults of endothelial activation, fibrinolysis, and consumption of platelets and coagulation factors, all aggravating an already attenuated hematological profile. ${ }^{50}$

Several documents and guidelines have been established with regards to treatment of patients on oral anticoagulants presenting with an acute bleed, or in the perioperative setting of emergency surgeries, mainly non-cardiac, where correction of the coagulopathy typically takes place before the incision is made. ${ }^{20,51}$ Cardiac surgery patients, on the other hand, present a unique challenge, especially in an emergency setting, where answers to some of the following questions may shed some light on the dilemma and aggressiveness in attempting to correct an underlying coagulopathy:

1-Does the preoperative administration of reversal agents hamper subsequent heparinization and achievement of therapeutic ACT needed for CPB? If so, when is the best time to administer, pre or post pump? While a normal coagulation profile is initially desirable to ensure a dry entry and minimize blood loss, the patient will paradoxically require full anticoagulation shortly thereafter for initiation of CPB. Therefore, it is paramount that any treatment modalities instituted to correct the coagulopathy do not interfere with the ability to fully and rapidly anticoagulate with heparin in preparation for CPB. Reports have emerged on the difficulty of adequately anticoagulating patients with heparin required for $\mathrm{CPB}$ or even during endovascular repair procedures following the administration of andexanet alfa that has led to heparin resistance. ${ }^{33}$ This is due to the binding of andexanet alfa to heparin-antithrombin complexes preventing them from exerting their anticoagulant function. Higher doses of heparin or antithrombin supplementation maybe required to achieve therapeutic anticoagulation. ${ }^{31,32}$

Therefore, pre CPB administration of specific reversal antidotes in a situation of primary sternotomy for emergency CABG, where minimal tissue dissection is anticipated, heparin resistance might be problematic if quick initiation of $\mathrm{CPB}$ is needed. On the other hand, redo-sternotomy cases require meticulous tissue dissection and a "dryer" operative field, where pre CPB correction of underlying coagulopathy would be desirable. Surgical judgment will dictate the need of full or partial correction of coagulopathy depending on the anticipated amount of dissection, realizing that heparin resistance may become an issue later on.

2-What is the risk of thromboembolism? Attention is generally directed towards avoiding bleeding, but discussion is not complete without noting the risk of thromboembolism secondary to anticoagulant interruption. ${ }^{8,9}$ Postoperative bleeding will require longer delay in the resumption of the anticoagulant with the potential of increased postoperative thromboembolic risk. While achieving near normal lab values is desirable, the reversal agents may have the unwanted effect of causing thrombosis, and their impact on improving outcomes is not well established. ${ }^{24,30}$ However, the thromboembolic rate with PCC treatment was noted to be comparable with that of anticoagulation discontinuation without reversal. While the studies examining the reversal agents were prospective, they were open-labeled and lacked a control group. ${ }^{22,27}$ The timing of postoperative anticoagulant resumption should be based on the bleeding vs thrombotic risk according to the CHA2DS2-VASc Score. ${ }^{34}$

3-Is postoperative anticoagulation needed, as in cases with mechanical valves or post pump assist devices? These patients present additional challenges, where aggressive correction of coagulopathy may lead to valve or device circuit thrombosis. The use of heparin-bonded tubing as well as biological rather than mechanical valves should be considered. Surgical judgment and patient's clinical condition will have to dictate proper course of action. For example, slight increase in chest tube drainage can be tolerated, and aggressive correction of lab values if patients are not bleeding is probably unnecessary and should be avoided.

4-Was the underlying indication for preoperative anticoagulation addressed at the time of surgery? An example would be concomitant MAZE and left atrial appendage (LAA) clipping for preexisting AF (STS 
Class I recommendation, evidence level A and B), where the risk of future postoperative embolization may be reduced such that the coagulopathy can be corrected and the need for postoperative anticoagulation avoided or at least delayed. ${ }^{52}$

Proposals in the literature on management of the cardiac surgical patient on anticoagulants in the acute setting are mainly based on expert opinion and extrapolation from non-emergent cardiac or emergent noncardiac guidelines, rather than from controlled trials. Proper knowledge of mechanism of action of the anticoagulants and the effectiveness as well as potential limitations of available reversal agents is important. These agents are costly and may lead to heparin resistance that could preclude timely institution of CPB; therefore, their judicious use is essential. Decision is typically made on a case-by-case basis considering product availability, local hospital policies and overall patient's clinical condition. The development of hospital and system-wide strategies that promote a multidisciplinary approach utilizing evidence-based clinical practices ensures appropriate and judicious use of these important resources.

\section{Conclusion:}

Currently, there are no randomized trials or well-designed observational studies that could direct the treatment of the difficult subset of patients on oral anticoagulants or antiplatelet medications requiring emergent cardiac surgery. In addition, the best metrics for complete resolution of anticoagulant effect in not always known or available for the newer DOACs. Therefore, at the present time only general recommendations can be made and used as guiding principles based on expert opinion that include the following:

1. Consider less invasive alternatives to surgery, such as PCI for ACS.

2. Delay surgery when possible.

3. Treat coexisting issues such as sepsis.

4. Avoid excessive hypothermia.

5. Exercise meticulous surgical techniques.

6. Correct underlying coagulopathy. Four-factor PPC can be used in patients on VKAs or DOACs, especially if CPB needs to be instituted quickly.

7. Specific reversal agents such as idarucizumab and andexanet alfa can be considered in cases where significant tissue dissection is anticipated such as redo sternotomy; however, this may lead to heparin resistance and anticoagulant rebound.

8. Cytosorb adsorption may be promising for patients on antiplatelet medications; otherwise, platelet transfusion might be necessary.

Patients on oral anticoagulants and antiplatelet medications requiring emergency cardiac surgery present unique and formidable treatment challenges. Benefits and risks of delaying or proceeding with surgical intervention should be carefully weighed, through an individualized heart-team approach. Treatment paradigms described above along with specific institutional guidelines, algorithms and policies for urgent reversal of anticoagulants are all helpful and should be developed to ensure best possible outcomes.

\section{Abbreviations:}

ACS: acute coronary syndrome

ACT: activated clotting time

AF: atrial fibrillation

CABG: coronary artery bypass grafting

CAD: coronary artery disease

CPB: cardiopulmonary bypass

DOAC: direct oral anticoagulation

FFP: fresh frozen plasma 
INR: international normalized ratio

VKA: vitamin-K antagonist

PCC: prothrombin complex concentrate

PCI: percutaneous coronary intervention

pRBC: packed red blood cell

STS: society of thoracic surgery

References:

1. Biancari F, Mikkola R, Heikkinen J, Lahtinen J, Airaksinen KEJ, Juvonen T. Estimating the risk of complications related to re-exploration for bleeding after adult cardiac surgery : a systematic review and meta-analysis. Eur J Cardiothorac Surg . 2012;41(1):50-55. doi:10.1016/j.ejcts.2011.04.023

2. Vivacqua A, Koch CG, Yousuf AM, Nowicki ER, Houghtaling PL, Blackstone EH, et al. Morbidity of Bleeding After Cardiac Surgery : Is It Blood Transfusion, Reoperation for Bleeding, or Both? Ann Thorac Surg . 2011;91(6):1780-1790. doi:10.1016/j.athoracsur.2011.03.105

3. Koch C, Li L, Duncan A, Mihaljevic T, Cosgrove D, Loop F. Morbidity and mortality risk associated with red blood cell and blood- component transfusion in isolated coronary artery bypass grafting. Crit Care Med . 2006;34(6):1608-1616. doi:10.1097/01.CCM.0000217920.48559.D8

4. Schumer EM, Chaney JH, Trivedi JR, Linsky PL, Williams ML, Slaughter MS. Emergency Coronary Artery Bypass Grafting: Texas Hear Inst J . 2016;43(3):214-219.

5. Mozaffarian D, Benjamin EJ, Go AS, Arnett DK, Blaha MJ, Cushman M, et al. AHA Statistical Update Heart Disease and Stroke Statistics - 2016 Update A Report From the American Heart Association. Circulation . 2016;133:38-360. doi:10.1161/CIR.0000000000000350

6. Barnes GD, Lucas E, Alexander GC, Goldberger ZD. National Trends in Ambulatory Oral Anticoagulant Use. Am J Med . 2019;128(12):1300-1305.e2. doi:10.1016/j.amjmed.2015.05.044

7. Glotzer T, Hellkamp A, Zimmerman J, Sweeney M, Yee R, Marinchak R. Atrial High Rate Episodes Detected by Pacemaker Diagnostics Predict Death and Stroke. Circulation . 2003;107:1614-1619.

8. Eikelboom J, Oldgren J, Ph D, Parekh A, Pogue J, Sc M, et al. Dabigatran versus warfarin in patients with atrial fibrillation. N Engl J Med . 2009;361(12):1139-1151.

9. Hylek EM, Hanna M, Al-khalidi HR, Ph D, Ansell J, Atar D, et al. Apixaban versus Warfarin in Patients with Atrial Fibrillation. $N$ Engl J Med . 2011;365(11):981-992.

10. Wallentin L, Becker R, A B, CP C. Ticagrelor versus clopidegrol in patients with acute coronary syndrome. N Engl J Med . 2009;361:1045-1057. doi:10.1056/NEJMoa1505949

11. Shih AW, Crowther MA. Reversal of direct oral anticoagulants: a practical approach. Hematol Am Soc Hematol Educ Progr . 2016;2(1):612-619.

12. Scaglione F. New Oral Anticoagulants: Comparative Pharmacology with Vitamin K Antagonists. Clin Pharmacokinet . 2013;52(2):69-82. doi:10.1007/s40262-012-0030-9

13. Antovic JP, Skeppholm M, Eintrei J, Boija EE, Söderblom L, Norberg E, et al. Evaluation of coagulation assays versus LC-MS / MS for determinations of dabigatran concentrations in plasma. Eur J Clin Pharmacol . 2013;69(11):1875-1881. doi:10.1007/s00228-013-1550-4

14. Favaloro E, Bonar R, Butler J, Marsden K. Laboratory testing for the new oral anticoagulants: a review of current practice. Pathology . 2013;45(4):435-437. 
15. Reilly P, Lehr T, Haertter S, Connolly S, Yusuf S, Eikelboom J, et al. The Effect of Dabigatran Plasma Concentrations and Patient Characteristics on the Frequency of Ischemic Stroke and Major Bleeding in Atrial Fibrillation Patients: the RE-LY Trial (Randomized Evaluation of Long-Term Anticoagulation Therapy). $J$ Am Coll Cardiol . 2014;63(4):321-328. doi:10.1016/j.jacc.2013.07.104

16. Nowak G. The ecarin clotting time, a universal method to quantify direct thrombin inhibitors. Pathophysiol Haemost Thromb . 2003;33(4):173-183. doi:10.1159/000081505

17. Samuelson BT, Cuker A. Blood Reviews Measurement and reversal of the direct oral anticoagulants. Blood Rev . 2019;31(1):77-84. doi:10.1016/j.blre.2016.08.006

18. Seyve L, Richarme C, Polack B, Marlu R. Impact of four direct oral anticoagulants on rotational thromboelastometry (ROTEM). Int J Lab Hematol . 2018;40(1):84-93. doi:10.1111/ijlh.12744

19. Zghaibe W, Scheuermann S, Munting K, Blaudszun G, Besser M, Ortmann E, et al. Clinical utility of the Quantra@ point-of-care haemostasis analyser during urgent cardiac surgery. Anaesthesia . 2020;75(3):366373. doi:10.1111/anae.14942

20. Tomaselli GF, Mahaffey KW, Cuker A, Dobesh PP, Doherty JU, Eikelboom JW, et al. 2020 ACC Expert Consensus Decision Pathway on Management of Bleeding in Patients on Oral Anticoagulants: A Report of the American College of Cardiology Solution Set Oversight Committee. J Am Coll Cardiol . 2020;76(5):594-622. doi:10.1016/j.jacc.2020.04.053

21. Grottke O, Aisenberg J, Bernstein R, Goldstein P, Huisman M V, Jamieson DG, et al. Efficacy of prothrombin complex concentrates for the emergency reversal of dabigatran-induced anticoagulation. Crit Care . 2016;20:115. doi:10.1186/s13054-016-1275-8

22. Majeed A, Ågren A, Holmström M, Bruzelius M, Chaireti R, Odeberg J, et al. Management of rivaroxaban- or apixaban-associated major bleeding with prothrombin complex concentrates: A cohort study. Blood . 2017;130(15):1706-1712. doi:10.1182/blood-2017-05-782060

23. Goldstein JN, Refaai MA, Jr TJM, Lewis B, Goldberg-alberts R, Hug BA, et al. Four-factor prothrombin complex concentrate versus plasma for rapid vitamin $\mathrm{K}$ antagonist reversal in patients needing urgent surgical or invasive interventions : a phase 3b , open-label, non-inferiority, randomised trial. Lancet . 2015;385(9982):2077-2087. doi:10.1016/S0140-6736(14)61685-8

24. Demeyere R, Gillardin S, Arnout J, Strengers PFW. Comparison of fresh frozen plasma and prothrombin complex concentrate for the reversal of oral anticoagulants in patients undergoing cardiopulmonary bypass surgery: a randomized study. Int J Transfus Med . 2010;99(3):251-260. doi:10.1111/j.14230410.2010.01339.x

25. Myles PS, Smith JA, Forbes A, Silbert B, Jayarajah M, Painter T, et al. Tranexamic Acid in Patients Undergoing Coronary-Artery Surgery. $N$ Engl J Med . 2017;376(2):136-148. doi:10.1056/nejmoa1606424

26. Cuker A, Burnett A, Triller D, Crowther M, Ansell J, Cott EM Van, et al. Reversal of direct oral anticoagulants: Guidance from the Anticoagulation Forum. Am J Hematol . 2019;94(6):697-709. doi:10.1002/ajh.25475

27. Pollack C, Reilly P, van Ryn J, Eikelboom J, Glund S, Bernstein R. Idarucizumab for Dabigatran Reversal — Full Cohort Analysis. N Engl J Med . 2017;377(5):431-441. doi:10.1056/NEJMoa1707278

28. Crespo-leiro MG, Amador RL, Granados L, Barge-caballero SMCDE, Segovia-cubero J, Rangel-sousa FGD, et al. Use of Idarucizumab to reverse the anticoagulant effect of dabigatran in cardiac transplant surgery . A multicentric experience in Spain. Clin Transpl . 2019;33(12):e13748. doi:10.1111/ctr.13748

29. Connolly SJ, Crowther M, Eikelboom JW, Gibson CM, Curnutte JT, Lawrence JH, et al. Full study report of andexanet alfa for bleeding associated with factor Xa inhibitors. N Engl J Med . 2019;380(14):13261335. doi:10.1056/NEJMoa1814051 
30. Schulman S, Gross PL, Ritchie B, Nahirniak S, Lin Y, Lieberman L, et al. Prothrombin Complex Concentrate for Major Bleeding on Factor Xa Inhibitors : A Prospective Cohort Study. Thromb Haemost . 2018;118:842-851.

31. Watson CJ, Zettervall S, Hall M, Ganetsky M. Difficult Intraoperative Heparinization Following Andexanet Alfa Administration. Clin Pract Cases Emerg Med . 2019;3(4):390-394. doi:10.5811/cpcem.2019.9.43650

32. Flaherty D, Connors JM, Singh S, Sylvester KW, Rimsans J, Cornella L. Andexanet Alfa for Urgent Reversal of Apixaban Before Aortic Surgery Requiring Cardiopulmonary Bypass: A Case Report. A\&BA Pract . 2019;13(7):271-273. doi:10.1213/xaa.0000000000001052

33. Levy JH, Welsby I. Andexanet Alfa Use in Patients Requiring Cardiopulmonary Bypass: Quo Vadis? AESA Pract . 2019;13(12):477-477. doi:10.1213/xaa.0000000000001115

34. Erdoes G, De Arroyabe B, Bolliger D, Ahmed A, Koster A, Agarwal S, et al. International consensus statement on the peri-operative management of direct oral anticoagulants in cardiac surgery.Anaesthesia . 2018;73(12):1535-1545.

35. Parasrampuria DA, Marbury T, Matsushima N, Chen S, Wickremasingha PK, He L, et al. Pharmacokinetics, safety, and tolerability of edoxaban in end-stage renal disease subjects undergoing haemodialysis. Thromb Haemost . 2015;113(4):719-727. doi:10.1160/TH14-06-0547

36. Awtry E, Loscalzo J. Aspirin. Circulation . 2000;101(10):1206-1218. doi:10.1097/01.NURSE.0000524762.35753.23

37. Myles PS, Smith JA, Forbes A, Silbert B, Jayarajah M, Painter T, et al. Stopping vs. continuing aspirin before coronary artery surgery. $N$ Engl J Med . 2016;374(8):728-737. doi:10.1056/NEJMoa1507688

38. Jacob M, Smedira N, Blackstone E, Williams S, Cho L. Effect of timing of chronic preoperative aspirin discontinuation on morbidity and mortality in coronary artery bypass surgery. Circulation . 2011;123(6):577583. doi:10.1161/CIRCULATIONAHA.110.957373

39. Hansson EC, Malm CJ, Hesse C, Bjorn Hornestam, Dellborg M, Rexius H, et al. Platelet function recovery after ticagrelor withdrawal in patients awaiting urgent coronary surgery. Eur $J$ Cardio-thoracic Surg . 2017;51(4):633-637. doi:10.1093/ejcts/ezw373

40. Hassan K, Kannmacher J, Wohlmuth P, Budde U, Schmoeckel M, Geidel S. Cytosorb Adsorption During Emergency Cardiac Operations in Patients at High Risk of Bleeding. Ann Thorac Surg . 2019;108(1):45-51. doi:10.1016/j.athoracsur.2018.12.032

41. Ranucci M, Bozzetti G, Ditta A, Cotza M, Carboni G, Ballotta A, et al. Surgical Reexploration After Cardiac Operations : Why a Worse Outcome? Ann Thorac Surg . 2008;86(5):1557-1562. doi:10.1016/j.athoracsur.2008.07.114

42. Frojd V, Jeppsson A. Reexploration for Bleeding and Its Association With Mortality After Cardiac Surgery. Ann Thorac Surg . 2016;102(1):109-117. doi:10.1016/j.athoracsur.2016.01.006

43. Mehta R, Sheng S, O'Brien S, Grover F, Gammie J, Ferguson T, et al. Reoperation for bleeding in patients undergoing coronary artery bypass surgery: incidence, risk factors, time trends, and outcomes. Circ Cardiovasc Qual Outcomes . 2009;2(6):583-590.

44. Biancari F, Airaksinen KEJ, Lip GYH. Benefits and risks of using clopidogrel before coronary artery bypass surgery: Systematic review and meta-analysis of randomized trials and observational studies. $J$ Thorac Cardiovasc Surg . 2012;143(3):665-675.e4. doi:10.1016/j.jtcvs.2011.01.069

45. Mannacio V, Meier P, Antignano A, Di Tommaso L, De Amicis V, Vosa C. Individualized strategy for clopidogrel suspension in patients undergoing off-pump coronary surgery for acute coronary syndrome: A case-control study. J Thorac Cardiovasc Surg . 2014;148(4):1299-1306. doi:10.1016/j.jtcvs.2013.12.011 
46. Stone GW, Clayton TC, Mehran R, Dangas G, Parise H, Fahy M. Impact of major bleeding and blood transfusions after cardiac surgery: Analysis from the Acute Catheterization and Urgent Intervention Triage strategY (ACUITY) trial. Am Heart J . 2012;163(3):522-529. doi:10.1016/j.ahj.2011.11.016

47. Kapetanakis EI, Medlam DA, Boyce SW, Haile E, Hill PC, Dullum MKC, et al. Clopidogrel administration prior to coronary artery bypass grafting surgery: The cardiologist's panacea or the surgeon's headache? Eur Heart $J$. 2005;26(6):576-583. doi:10.1093/eurheartj/ehi074

48. Ferraris VA, Brown JR, Despotis GJ, Hammon JW, Reece TB, Saha SP, et al. 2011 Update to The Society of Thoracic Surgeons and the Society of Cardiovascular Anesthesiologists Blood Conservation Clinical Practice Guidelines * The Society of Thoracic Surgeons Blood Conservat. Ann Thorac Surg . 2011;91(3):944982. doi:10.1016/j.athoracsur.2010.11.078

49. Valeri C, Khabbaz K, Sf K, Marquardt C, Ragno G, Feingold H, et al. Effect of skin temperature on platelet function in patients undergoing extracorporeal bypass. J Thorac Cardiovasc Surg . 1992;104(1):108116.

50. Despotis GJ, Avidan MS, Hogue CW. Mechanisms and Attenuation of Hemostatic Activation During Extracorporeal Circulation. Ann Thorac Surg . 2001;72(5):S1821-31.

51. Levy JH. Discontinuation and management of direct-acting anticoagulants for. Am J Emerg Med . 2019;34(11):14-18. doi:10.1016/j.ajem.2016.09.048

52. Badhwar V, Rankin JS, Damiano RJ, Gillinov AM, Bakaeen FG, Edgerton JR, et al. The Society of Thoracic Surgeons 2017 Clinical Practice Guidelines for the Surgical Treatment of Atrial Fibrillation. Ann Thorac Surg . 2017;103(1):329-341. doi:10.1016/j.athoracsur.2016.10.076

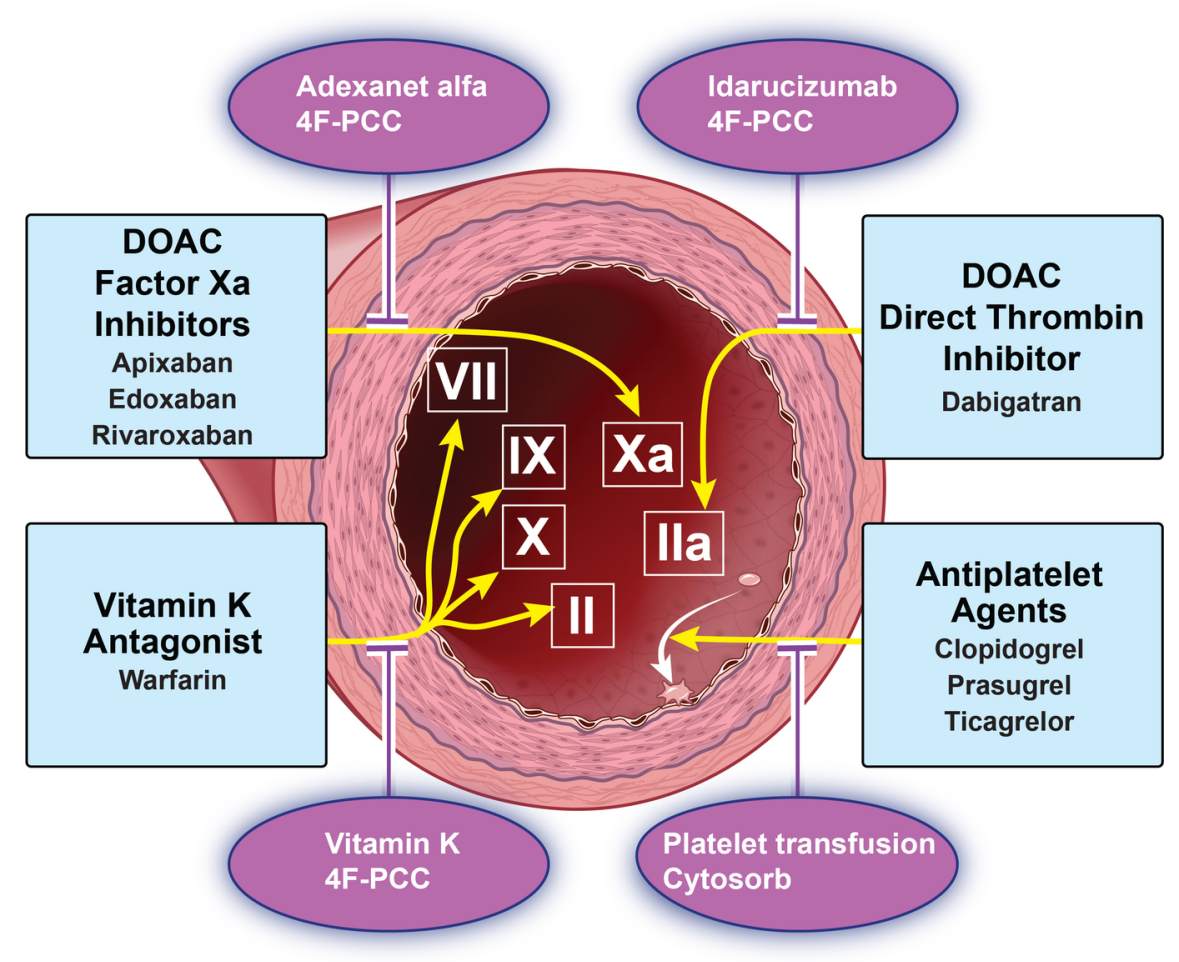




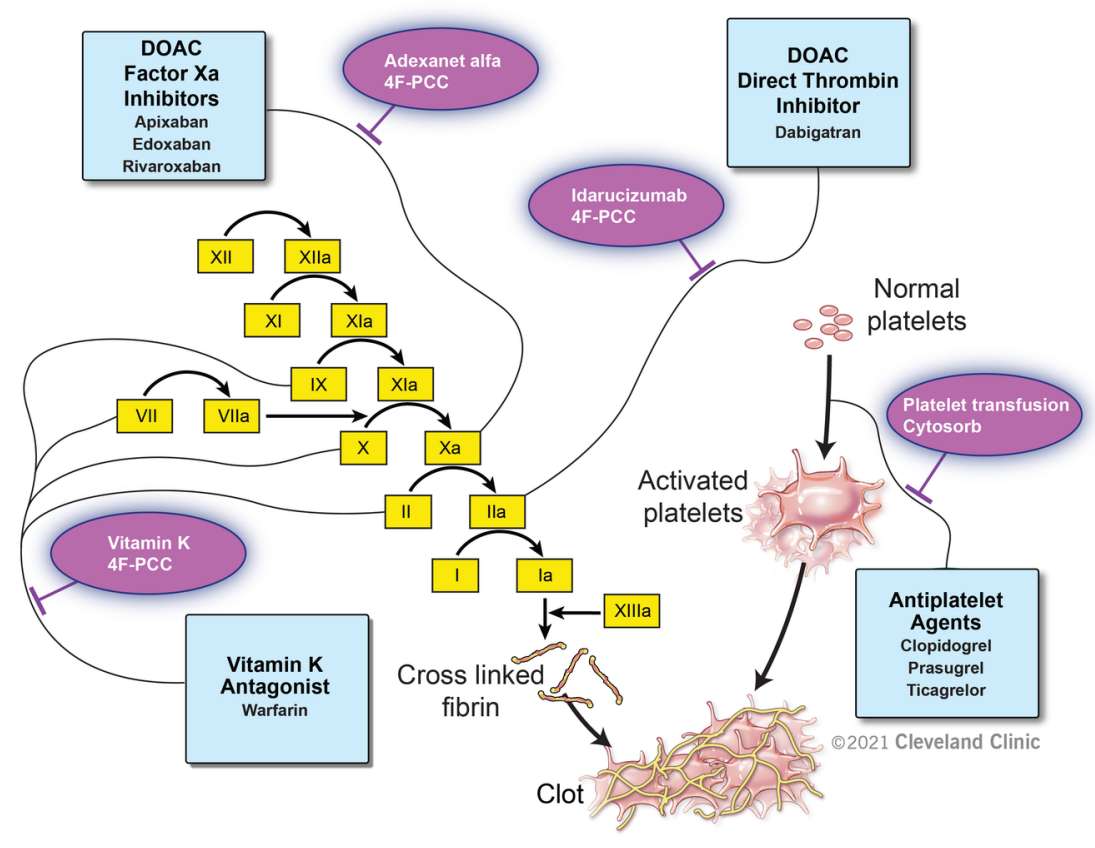

\section{Hosted file}

table 1.docx available at https://authorea.com/users/322631/articles/523209-emergencycardiac-surgery-in-patients-on-oral-anticoagulants-and-antiplatelet-medications

\section{Hosted file}

table 2 .docx available at https://authorea.com/users/322631/articles/523209-emergencycardiac-surgery-in-patients-on-oral-anticoagulants-and-antiplatelet-medications

\section{Hosted file}

table 3.docx available at https://authorea.com/users/322631/articles/523209-emergencycardiac-surgery-in-patients-on-oral-anticoagulants-and-antiplatelet-medications 